This is the accepted manuscript made available via CHORUS. The article has been published as:

Sequential Activation of Molecular Breathing and Bending during Spin-Crossover Photoswitching Revealed by Femtosecond Optical and X-Ray Absorption Spectroscopy Marco Cammarata, Roman Bertoni, Maciej Lorenc, Hervé Cailleau, Sergio Di Matteo, Cindy Mauriac, Samir F. Matar, Henrik Lemke, Matthieu Chollet, Sylvain Ravy, Claire Laulhé, Jean-François Létard, and Eric Collet

Phys. Rev. Lett. 113, 227402 - Published 26 November 2014 DOI: 10.1103/PhysRevLett.113.227402 


\section{Sequential activation of molecular breathing and bending during spin-crossover photoswitching revealed with femtosecond optical and $x$-ray absorption spectroscopy}

Marco Cammarata, ${ }^{1}$ Roman Bertoni, ${ }^{1}$ Maciej Lorenc,,${ }^{1}$ Hervé Cailleau,,${ }^{1}$ Sergio Di Matteo,,${ }^{1}$ Cindy Mauriac, ${ }^{2}$ Samir Matar, ${ }^{2}$ Henrik Lemke, ${ }^{3}$ Matthieu Chollet, ${ }^{3}$ Sylvain Ravy, ${ }^{4}$ Claire Laulhé, ${ }^{4}$ JeanFrançois Létard, ${ }^{2}$ Eric Collet, ${ }^{1, a}$

${ }^{1}$ Institut de Physique de Rennes, UMR CNRS 6251Université Rennes 1, 35042 Rennes cedex, France.

${ }^{2}$ CNRS, Université de Bordeaux, ICMCB, 87 avenue du Dr. A. Schweitzer, Pessac, 33608 France.

${ }^{3}$ LCLS, SLAC National Laboratory, Menlo Park, CA, USA.

${ }^{4}$ Synchrotron SOLEIL, L'Orme des Merisiers, Saint Aubin, 91192 Gif-sur-Yvette France.

We study the basic mechanisms allowing light to photoswitch at molecular scale a spin-crossover material from low- to high-spin state. Combined femtosecond x-ray absorption performed at LCLS $\mathrm{X}$-FEL and optical spectroscopy reveal that the structural stabilization of the photoinduced HS state results from a two step structural trapping. Molecular breathing vibrations are first activated and rapidly damped as part of the energy is sequentially transferred to molecular bending vibrations. During the photoswitching, the system follows a curved trajectory on the potential energy surface.

PACS Codes : 42.70.Gi, 75.30.Wx, 78.47.J-, 82.53.Xa

\footnotetext{
${ }^{a}$ To whom correspondence should be addressed. E-mail: eric.collet@univ-rennes1.fr
} 
Introduction. - A wide range of phenomena in matter are driven by changes that occur after illumination with light. ${ }^{1}$ This includes chemical, bio-chemical or solid-state reactions, ${ }^{2,3,4}$ as well as switching of functionality of materials such as conductivity ${ }^{5}$ or magnetism. ${ }^{6}$ During a transformation triggered by a femtosecond (fs) laser pulse the excited system is launched onto a complex pathway, from the initial photoexcited to the final photoinduced state, whereby nonequilibrium phenomena, coherent dynamics and interconversion of energy are interconnected. ${ }^{1}$ Transition metal complexes undergoing spin-crossover (SCO) from low-spin (LS) to high-spin (HS) state are prototypes of molecular photoswitching in materials. ${ }^{7}$ On the shortest time scales the molecule undergoes intersystem crossing (ISC) from the initial photoexcited LS- to HS-state. The structural reorganization of the ligand around the metal centre gives rise to an energy barrier between the trapped HS and the ground LS state. The initial mechanism behind the extremely fast ISC, between states that differ in both spin and structure, defy conventional descriptions. Recent theoretical studies by van Veenendaal ${ }^{8,9}$ on ISC in $\mathrm{Fe}^{\mathrm{II}} \mathrm{SCO}$, mediated by the spin-orbit coupling, discussed the role of significant changes of the metal-ligand distance (molecular breathing). It was proposed that the ultrafast ISC results from the dephasing of the photoexcited state into the HS phonon states. A monotonous decay of the photoexcited state to the HS state becomes efficient when this breathing mode is rapidly damped as energy is dissipated into the environment.

By combining femtosecond x-ray absorption near edge structure (XANES) and optical spectroscopy, we provide an experimental evidence of the ultrafast activation and damping of molecular breathing accompanying the ISC, which were lacking up to now. This process is followed by vibrational cooling in the HS potential observed on the bending mode. With respect to conventional models describing SCO photoswitching in terms of potential energy curve along a single breathing coordinate, our work reveals a more complex reality involving a curved trajectory, along breathing and bending, on a multi-dimensional potential energy surface.

Results. - The SCO $\left[\mathrm{Fe}(\text { phen })_{2}(\mathrm{NCS})_{2}\right]$ crystal investigated here, undergoes a first-order phase transition from $\mathrm{LS}\left(\mathrm{S}=0, t_{2 g}{ }^{6} e_{g}{ }^{0} L^{0}\right)$ to $\mathrm{HS}\left(\mathrm{S}=2, t_{2 g}{ }^{4} e_{g}{ }^{2} L^{0}\right)$ states above $\approx 180 \mathrm{~K}$ and exhibits photomagnetism and photochromism. ${ }^{7,10,11}$ Here, $L$ corresponds to the LUMO of the ligand. This molecular bi-stability is associated with important structural reorganizations around the $\mathrm{FeN}_{6}$ octahedron between both spin states (Fig. 1(a), S1 \& S2) ${ }^{12},{ }^{131415161718}$. The less bonding character of the HS state leads to the expansion of the average Fe-N bond length from $\langle F e-N\rangle_{L S}=1.97 \AA$ 
to $\langle\mathrm{Fe}-\mathrm{N}\rangle_{H S}=2.16 \AA$, often observed for $\mathrm{Fe}^{\mathrm{II}} \mathrm{SCO}{ }^{7,19}$. That changes the XANES spectrum as shown in Fig. 1b, in agreement with the published data on this molecular crystal. ${ }^{20}$

The ultrafast photoswitching dynamics of $\left[\mathrm{Fe}(\text { phen })_{2}(\mathrm{NCS})_{2}\right]$ is investigated in the LS phase at $140 \mathrm{~K}$ by two complementary pump-probe methods ${ }^{12} . \mathrm{A} \approx 50$ fs laser pulse $(650 \mathrm{~nm})$ photoswitches LS state to HS via a metal-to-ligand charge-transfer process (MLCT).$^{10}$ The changes in XANES are recorded with $\approx 30$ fs $\mathrm{x}$-ray pulses at the XPP station of the LCLS X-FEL, ${ }^{21}$ while changes in optical reflectivity (OR) and transmission (OT) are recorded with $\approx 50$ fs VIS-NIR spectroscopy. Fig. 2(a) shows the time course of the XANES signal measured at two energies most sensitive to the structural change (Fig. 1). The increase of XANES signal at $7.125 \mathrm{keV}$ and the decrease at $7.148 \mathrm{keV}$ mainly result from Fe-N elongation ${ }^{20}$ and are the fingerprints of the formation of HS structures. We fit the rising time by convolving a Gaussian temporal Instrument Response Function (IRF) with an exponential rise $\left(\tau_{\mathrm{Fe}-\mathrm{N}}\right)$. The $110(10)$ fs FWHM IRF, obtained by using a timing tool ${ }^{21}$ designed to synchronize the optical and the $\mathrm{x}$-ray laser pulses, allows an accurate determination of $\tau_{\mathrm{Fe}-\mathrm{N}}=170(20) \mathrm{fs}$.

Figs. 2(b-d) \& S4 show time traces of OR and OT (with 110 fs IRF) in different spectral zones, revealing two main steps: the onset of OR change or a peak in OT immediately after laser excitation, relaxing towards a plateau during the first 100 's fs. The increase of optical absorption on the plateau, which translates into a simultaneous decrease of OR and OT in all probed regions, is another fingerprint of the LS-to-HS photo-switching, also observed during the thermal LS-toHS conversion ${ }^{10}$ (see also Fig. S3). Density of state calculations ${ }^{22}$ explain the stronger absorption of the HS state around $760 \mathrm{~nm}(1.6 \mathrm{eV})$ by a decrease of the energy gap between $t_{2 g}$ and $e_{g}$ bands from $\approx 1.9 \mathrm{eV}(\mathrm{LS})$ to $\approx 1.6 \mathrm{eV}$ (HS), due to the molecular ligand field weakening (see also Fig. $\mathrm{S} 5)$. OT and OR data at $760 \mathrm{~nm}$ indicate that the gap narrowing in HS state, resulting from the Fe-N elongation, occurs within $\tau_{\mathrm{Fe}-\mathrm{N}}=140$ (20) fs. It correlates well with the 170 (20) fs time constant obtained by XANES.

The transient OT peak corresponds to the absorption of the photoexcited singlet ${ }^{1} \mathrm{MLCT}$ state $\left(t_{2 g}^{5} e_{g}^{0} L^{l}\right)$, and other possible intermediate (INT) electronic states ${ }^{9}$, and results from transition from a ligand level $L$ to higher energy states (see Fig. S5) ${ }^{10,12} .760 \mathrm{~nm}$ therefore probes both INT and HS $\left(t_{2 g}-e_{g}\right)$ absorptions. The $t_{2 g}-e_{g}$ absorption is no more accessible at $850 \mathrm{~nm}$ and above, and the main effect in the time traces is the absorption of the INT electronic state. This transient OT peak above $850 \mathrm{~nm}$ (Fig. 2(c-d) \& S4) better characterizes the depopulation of the INT state, 
which decays within less than $50 \mathrm{fs}$. Thus far, our results determine an ISC of tens of fs and a $\approx 160 \mathrm{fs}$ Fe-N bond elongation in a solid, both of which are similar to those reported for $\mathrm{Fe}^{\mathrm{II}}$ molecules in solution ${ }^{23,24,25,26,27,28}$.

On top of such changes, clear oscillating components are present in the optical data. Fig. 3a-c shows the residuals after exponential fit. Data at $760 \mathrm{~nm}$ show a coherent vibration around 113 $\mathrm{cm}^{-1}$ ( $\approx 300 \mathrm{fs}$ oscillation period) in the $[0-700 \mathrm{fs}]$ range. The corresponding time-dependent Fourier transforms are presented in Fig. 3(d-f) \& S4 and the apparent rising time of the $113 \mathrm{~cm}^{-1}$ mode is limited by the Gaussian temporal window used ${ }^{12}$. It was already discussed above that OT and $\mathrm{OR}$ at $760 \mathrm{~nm}$ are sensitive to the Fe-N elongation, hereafter referred to as the breathing coordinate $D$. Therefore, the oscillations riding on $760 \mathrm{~nm}$ signals reflect the breathing mode, i.e. the totally symmetric $\mathrm{Fe}-\mathrm{N}$ stretching, in relation with the symmetry conserving picture of the displacive spin-state switching. This observation is supported by calculations, according to which the only totally symmetric mode (symmetry $\mathrm{A}$ in the $\mathrm{C}_{2}$ point group of the complex) in this frequency range is the breathing mode $\left(\approx 125 \mathrm{~cm}^{-1}\right)$ in the HS state ${ }^{29,30}$. The breathing mode is represented by green arrows in Fig. 1 and shown in video $\mathrm{S} 1^{12}$. XANES data do not reveal such small oscillating features after the $\approx 0.2 \AA$ elongation, because of the noise limit of the measurements, but we underline that the main Fe-N elongation occurs on a timescale approaching the half period of the breathing mode (Fig. 4(a)).

Optical data at 550, $900 \mathrm{~nm}$ (Fig. 2(c)) and $950 \mathrm{~nm}$ (Fig. S4) reveal another coherent vibration, in-phase for all these probing wavelengths. These oscillations around $85 \mathrm{~cm}^{-1}$ correspond to the butterfly mode (also of A symmetry) ${ }^{29,30}$, which bends the ligand and the N-Fe-N angles without significantly changing Fe-N distances (see purple arrows in Fig. 1 \& video S2). This is a bending coordinate $\Sigma^{19,31}$ and such a coherent ligand vibration was also observed for another $\mathrm{Fe}^{\mathrm{II}} \mathrm{SCO}$ molecular system in solution. ${ }^{26}$ Unlike the breathing mode, we observed that the bending mode with $\approx 390 \mathrm{fs}$ period is only activated coherently during a second step, after $\approx 500 \mathrm{fs}$, with a maximum spectral weight around 1300 fs (Fig. $3 \&$ S4). This bending occurs well after the $160 \mathrm{fs}$ initial Fe-N elongation.

Such oscillations cannot be attributed to impulsive Raman process in the ground LS state because the LS breathing frequency is significantly higher $\left(156 \mathrm{~cm}^{-1}\right)^{29,30}$. In addition, there is no optical transitions from LS state to other excited states which can be probed in the 760-950 nm region (Fig. S5) and consequently the LS state is optically silent in our data. 
Discussion.- In SCO materials the Fe-N elongations during LS-to-HS conversion are very similar, but the ligand bending $\Sigma^{12,19,31}$ also plays an important role for the relative stability of LS and HS states. Hauser evidenced in the kinetic studies of HS $\rightarrow$ LS relaxation a breakdown of the single mode model and proposed to describe such systems by qualitatively splitting the reaction coordinate into the breathing and the bending modes ${ }^{32}$. Fig. 4(b) is a cartoon of a likely potential energy surface $(\mathrm{PES})$ of $\left[\mathrm{Fe}(\text { phen })_{2}(\mathrm{NCS})_{2}\right]$ in the breathing and bending $(D, \Sigma)$ coordinate space. At thermal equilibrium (see ref 13 and Fig. S2) the LS potential is centred at $\left(D_{L S}=1.97 \AA\right.$, $\left.\Sigma_{L S}=35^{\circ}\right)$ and the one of the HS state at $\left(D_{H S}=2.16 \AA, \Sigma_{H S}=65^{\circ}\right)$. The delayed activation of the bending $\Sigma$, with respect to breathing $D$ is schematically represented in Fig. 4(b) on $(D, \Sigma)$ PES from stable LS to stable HS locations.

XANES, OT and OR data at $760 \mathrm{~nm}$ give an average elongation $D_{\text {mean }}(\mathrm{t})$ with a $\approx 160$ fs time constant. OR at $900 \mathrm{~nm}$, no more sensitive to $D$ but only to the change of $\Sigma$, gives an average torsion $\Sigma_{\text {mean }}(t)$ with 250 fs time constant. We thus deduce from these different breathing and bending timescales a curved trajectory in the relevant coordinate space $(D, 2)$ on PES. If we simply assume that the photoinduced HS state is not significantly different from the HS structure at thermal equilibrium, we can plot a trajectory in Fig. 3(h). It indicates that around 1 ps the motion is mainly along $\Sigma$, also simultaneously manifested by the strong spectral weight of the bending mode (Fig. 3(e-f)). The coupling of instantly photo-activated phonons to other modes can drive major structural reorganization in solids. ${ }^{4,33,34}$ In the present case, the energy transfer between the two totally symmetric breathing $(D)$ to bending $(2)$ modes can be described by a linear coupling, with the classical equations of motion of coupled harmonic oscillators:

$$
\left\{\begin{array}{l}
\mu_{D} \ddot{D}+\gamma_{D} \dot{D}+k_{D} D+k_{D \Sigma}(D-\Sigma)=0 \\
\mu_{\Sigma} \ddot{\Sigma}+\gamma_{\Sigma} \dot{\Sigma}+k_{\Sigma} \Sigma-k_{D \Sigma}(D-\Sigma)=0
\end{array}\right.
$$

$\mu_{i}$ are the reduced masses of the oscillators (obtained from Gaussian calculations ${ }^{12}$ ), $k_{i}$ are the force constants refined to reproduce 113 and $85 \mathrm{~cm}^{-1}$ frequencies of the oscillations, $\gamma_{i} / \mu_{i}$ are the damping rates and $k_{D \Sigma}$ is the coupling between the two modes. A numerical fit the oscillating part of the time dependent data ${ }^{12}$ with these equations of motion gives the time evolution of the oscillating components $D_{o s c}(\mathrm{t})$ and $\Sigma_{o s c}(\mathrm{t})($ Fig. $3(\mathrm{~g}))$. These curves reproduce well the initial activation of the $113 \mathrm{~cm}^{-1}$ breathing oscillation, in agreement with the displacive description of the ISC along $D$. The fit also evidence a very fast damping of the breathing mode within 166 fs 
$\left(\gamma_{D} / \mu_{D}=6 \mathrm{ps}^{-1}\right)$. These results are in agreement with the theoretical model ${ }^{8}$ introduced above, which considers an ISC of tens of femtoseconds accompanied by a structural elongation of the order of 100-200 fs and a damping time constant shorter than the period of the breathing mode (300 fs here). The observed delayed coherent activation of $\Sigma$ characterizes the energy transfer from the breathing to the bending modes schematically represented in Fig. $4 \mathrm{~b}$ and the smaller damping of the bending mode $\left(\gamma_{\Sigma} / \mu_{\Sigma}=1.16 \mathrm{ps}^{-1}\right)$ indicates that the system oscillates (in the HS potential) as it gets vibrationally cooled within $900 \mathrm{fs}$. The additional transfer to other modes, for instance to lower frequency optical lattice phonons, can also be considered. A lattice mode is observed at $33 \mathrm{~cm}^{-1}$ (Fig. 3d), but its spectral weight is weaker. Thus, the major part of energy of the absorbed photon $(1.9 \mathrm{eV})$ is dissipated, since each coherent molecular phonon only accounts for a small fraction of that energy. For instance, the energy of the breathing mode, $E=1 / 2 m \omega^{2} D^{2}$, can be estimated to only $40 \mathrm{meV}$, with reduced mass $m=8.5 \mathrm{amu}$, angular frequency $\omega=2 \pi * 113 \mathrm{~cm}^{-1}$ and elongation $D \approx 0.2 \AA$.

Conclusion. - In the emerging field of control science, understanding the physical processes allowing functionalization with light on ultrafast time scale is a key issue. Our results confirm the fast intersystem crossing observed in such transition-metal systems ${ }^{23-28}$, as the change of electronic state is shorter than $50 \mathrm{fs}$ and followed by a significant metal-ligand elongation within 160 fs. This initial structural change is directly coupled to the change of electronic state because of the less bonging nature of the HS state. Our experimental studies more importantly provides an experimental demonstration that the fast and highly efficient ISC is driven by the dephasing of the photoexcited state into the HS phonon states. The activated breathing mode is damped on a timescale (160 fs) close to its half-period, which allows an efficient trapping in the HS state as the decrease of the oscillation amplitude hinders recurrence to the initial state. We also show that the molecular bending is also involved, since the Fe-N elongation once established triggers the increase of the N-Fe-N bending because of ligand rigidity. Its delayed activation reveals a curved trajectory on a multi-dimensional potential energy surface, which underlines that the limits of conventional descriptions along a single breathing coordinate on potential energy curves. This description of the photoswitching at the molecular scale, accompanied by important energy redistribution, sets the initial conditions for the slower transformation at material scale ${ }^{35,36}$. Such 
combination of x-ray and optical spectroscopies is key to disentangle the role of different degrees of freedom of electronic and/or structural nature in photoinduced phenomena.

This work was supported by the Institut Universitaire de France, Rennes Métropole, Région Bretagne (CREATE 4146), ANR (ANR-13-BS04-0002), CNRS (PEPS SASLELX) and Europe (FEDER). Portions of this research were carried out at the Linac Coherent Light Source (LCLS) at SLAC National Accelerator Laboratory. LCLS is an Office of Science User Facility operated for the U.S. Department of Energy Office of Science by Stanford University. 


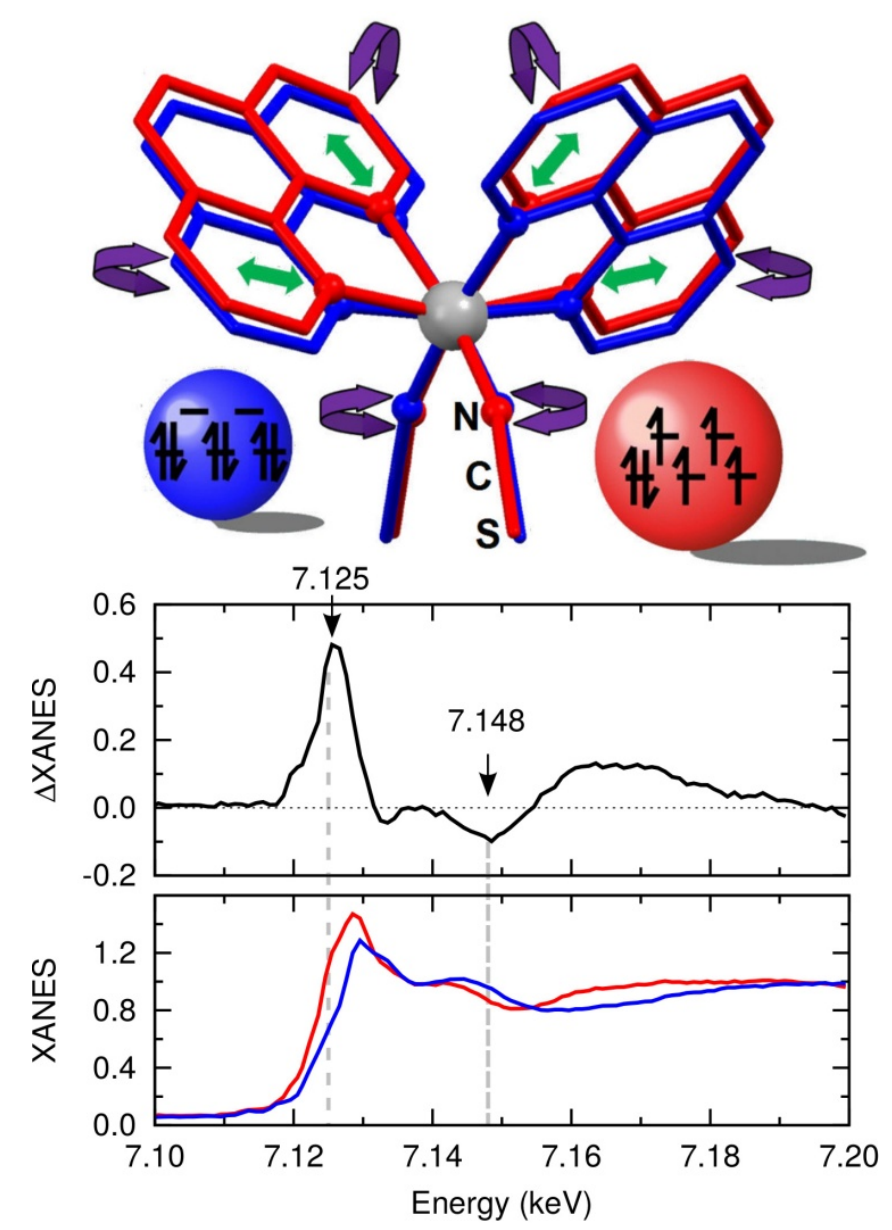

Fig. 1 (color online). (a) LS (140 K, blue) and HS (200 K, red) structures of $\left[\mathrm{Fe}(\text { phen })_{2}(\mathrm{NCS})_{2}\right]$ (phen=1,10-phenanthroline). The central Fe atom is bounded by N to the phenantroline and NCS groups. Green arrows represent the breathing mode and purple ones the bending mode. b) XANES spectra and difference $\triangle X A N E S$ measured between the LS and HS states. 
a)

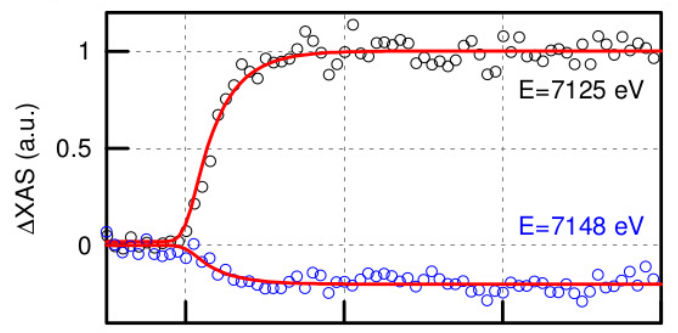

b)

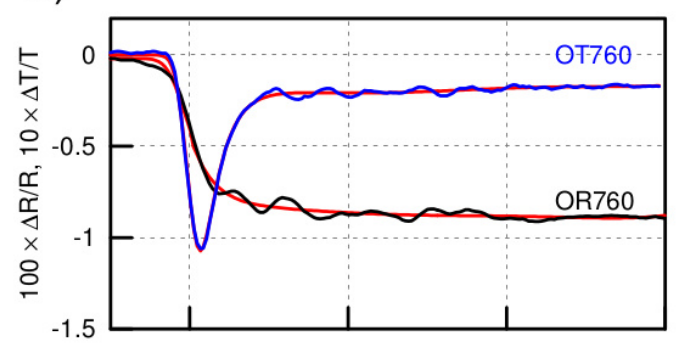

c)



d)

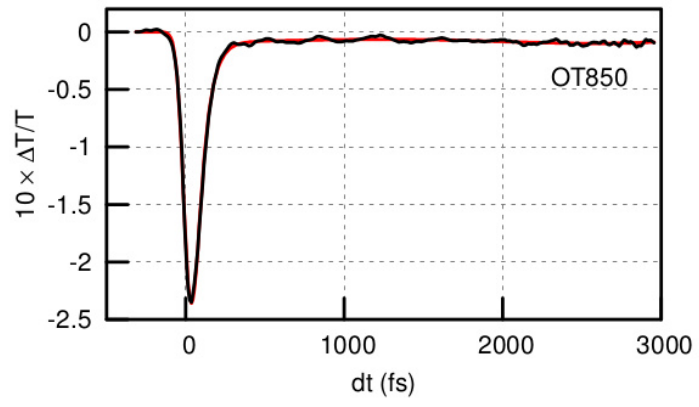

Fig. 2 (color online). Kinetic traces of XANES at 7125 and $7148 \mathrm{eV}$, fit with a singleexponential function of the rising edge $\left(\tau_{\mathrm{Fe}-\mathrm{N}}=170(10)\right.$ fs) represented by red solid lines (a). Kinetic traces of OR and OT at $760 \mathrm{~nm}$ (b), OR at $900 \mathrm{~nm} \& 550 \mathrm{~nm}$ and OT at $900 \mathrm{~nm}$ (c) and OT $850 \mathrm{~nm}$ (d). Fits by a single-exponential function describing the fast change plus a fifth order polynomial are represented by red solid lines. 
a)

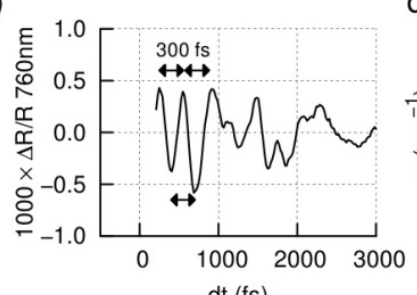

b)

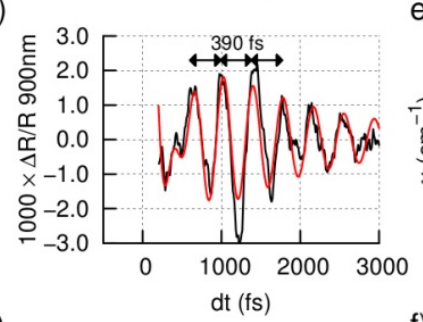

c)

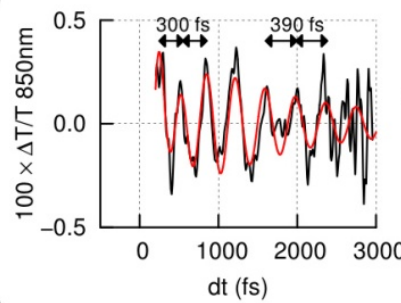

g)

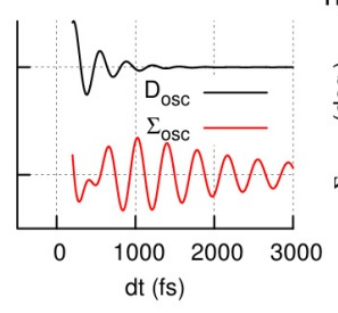

d)

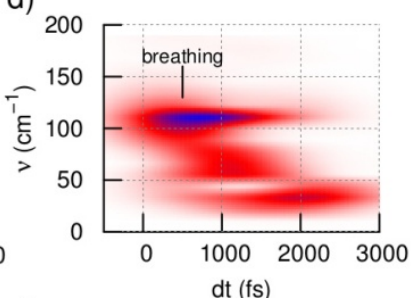

e)

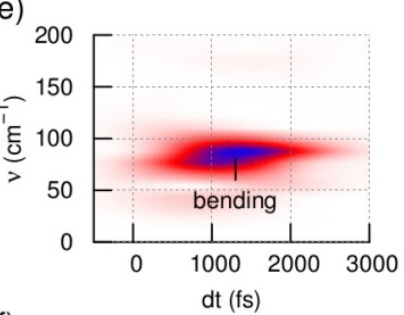

f)

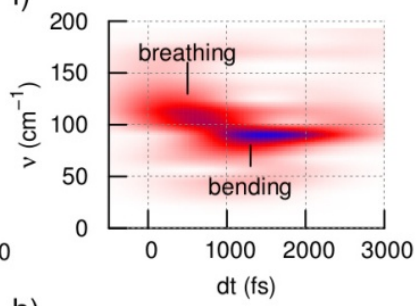

h)

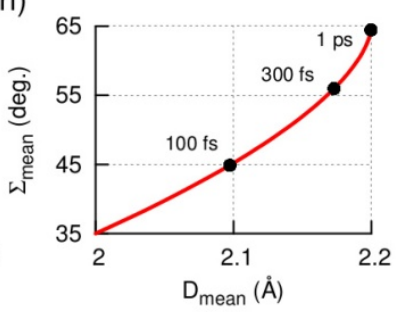

Fig. 3 (color online). (a-c), oscillating component of $O R$ at $760 \mathrm{~nm}, O R$ at $900 \mathrm{~nm}$ and $O T$ at $850 \mathrm{~nm}$. (d-f), time dependent FFT of the experimental data, showing the activation of the breathing mode and the delayed activation of the bending mode. Combined fits of OR at $900 \mathrm{~nm}$ and $\mathrm{OT}$ at $850 \mathrm{~nm}$ (red line) by the coupled oscillator model, which show the

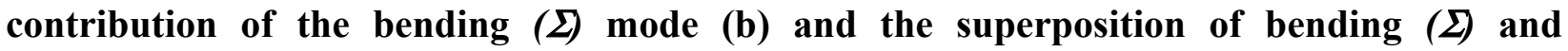
breathing $(D)$ modes $(c)$. The time course of the oscillating component $D_{\text {osc }}(300 \mathrm{fs})$ and $\Sigma_{o s c}(390 \mathrm{fs})$ obtained by the fit in (b) and (c) are displayed in (g) and the average evolutions of $D_{\text {mean }}$ and $\Sigma_{\text {mean }}$ obtained by the fit in Fig. 3 are displayed in (h). 


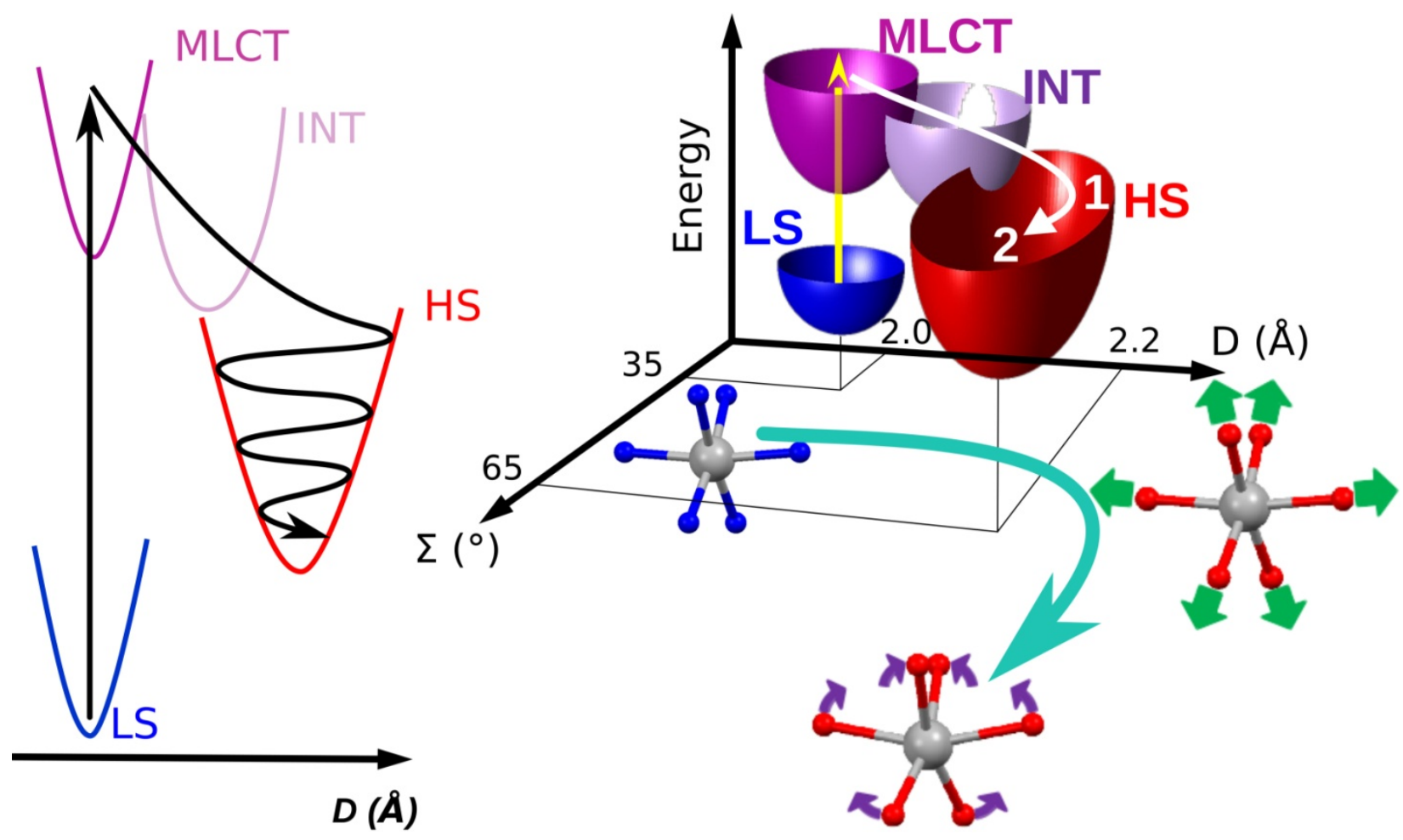

Fig. 4 (color online). (a) schematic representation of the elongation and damping of the breathing mode along the $D$ coordinate. (b) Classical trajectory in the $(D, Z)$ space. Molecules in the LS (blue) potential reach ${ }^{1}$ MLCT state by light excitation. Fast ISC, through possible bypassed INT states, drives $D$ elongation during step 1 with the generation and damping of breathing phonon, followed by activation of additional bending phonons such as distortion $\Sigma$, during step 2 . The sequence is sketched at the bottom. 
1 K. Nasu, Ed., Photoinduced phase transitions (Ed. World Scientist, Singapore, 2004).

2 V. S. Petrovic et al, Phys. Rev. Lett. 108, 253006 (2012).

3 D. Polli et al, Nature 467, 440-443 (2010).

4 H. Uemura, H. Okamoto. Phys. Rev. Lett. 105, 258302 (2010).

5 M. Gao et al, Nature 496, 343-346 (2013).

6 A. Kirilyuk, A.V. Kimel, T. Rasing. Rev. Mod. Phys. 82, 2731-2784 (2010).

7 M. Halcrow, Ed., Spin-crossover materials (Wiley, West Sussex, 2013) ISBN 9781119998679.

8 M. van Veenendaal, J. Chang, A.J. Fedro. Phys. Rev. Lett. 104, 067401 (2010).

9 J. Chuang, A.J. Fedro, M. van Veenendaal. Phys. Rev. B 82, 075124. (2010).

10 E. König, K. Madeja. Inorg. Chem. 6, 48 (1967).

11 E.W. Müller, H. Spiering, P. Gütlich. Chem. Phys. Lett. 93, 567 (1982).

12 See Supplemental Material [url], which includes Refs. [13-18].

13 J.A. Real, B. Gallois, T. Granier, F. Suez-Panama, J. Zarembovitch. Inorg. Chem. 31, 4972 (1992).

14 P. Ganguli, P., Gütlich, P. \& Mûller, E. W. Effect of Metal dilution on the Spin-crossover behavior on $\left[\mathrm{Fe}_{\mathrm{x}} \mathrm{M}_{1-\mathrm{x}}(\mathrm{phen})_{2}(\mathrm{NCS})_{2}\right]$. Inorg. Chem. 21, 3429-3433 (1982).

15 Frisch, M. J. et al, GAUSSIAN03 (revision C.02), Gaussian, Inc., Wallingford CT, 2004.

16 S. Matar, J.F. Létard. Z. Naturforsch 65b, $565-570$ (2010).

17 E. Jones, T. Oliphant, P. Peterson and others, SciPy: Open Source Scientific Tools for Python (2001-), http://www.scipy.org/.

$18 \mathrm{http}: / /$ wwwasdoc.web.cern.ch/wwwasdoc/minuit/minmain.html

19 M. Marchivie et al, J. Am. Chem. Soc. 124 194-195. (2002).

20 V. Briois et al, J. Am. Chem. Soc. 117, 1019-1026 (1995).

21 M. Harmand et al, Nat. Photonics 7, 215-218 (2013).

22 S. Matar, J.F. Létard. Z. Naturforsch 65b, 565 - 570 (2010).

23 C. Bressler et al, Science 323, 489-492 (2009).

24 N. Huse et al, J. Phys. Chem. Lett. 2, 880-884. (2011).

25 H.T. Lemke, et al, J. Phys. Chem. A 117, 735-740 (2013).

26 C. Consani et al, Angew. Chem. Int Ed. 121, 7320 (2009).

27 W. Gawelda et al, J. Am. Chem. Soc. 129, 8199-8206 (2007).

28 M. Chergui in Spin-crossover materials, M. Halcrow, Ed. (Wiley, West Sussex, 2013) ISBN 9781119998679.

29 G. Baranovic, D. Babic. Spectrochem. Acta A 60, 1013. (2004).

30 K.L. Ronayne et al, Phys. Chem. Chem. Phys. 8, 4685 (2006).

31 M. Buron-Le Cointe et al, Phys. Rev. B 85, 064114 (2012).

32 A. Hauser et al, Coord. Chem. Rev. 250, 1642-1652 (2006).

33 Y. Kawakami et al, Phys. Rev. Lett. 103, 066403. (2009).

34 M. Först et al, Nature Phys. 7, 854 (2011).

35 M. Lorenc et al, Phys. Rev. Lett. 103, 028301 (2009).

36 M. Lorenc et al, Phys. Rev. B. 85, 054302 (2012). 